\title{
Patient considerations in the management of ulcerative colitis: role of once-daily MMX mesalamine
}

\author{
Daniel B Zandman \\ Mark A Peppercorn \\ Harvard Medical School, Division \\ of Gastroenterology, Department \\ of Medicine, Beth Israel Deaconess \\ Medical Center, Boston, MA, USA
}

Correspondence: Mark A Peppercorn Harvard Medical School, Division of Gastroenterology, Department of Medicine, Beth Israel Deaconess Medical Center, 330 Brookline Avenue, Boston, MA 022I5, USA

Email mpepperc@bidmc.harvard.edu

\begin{abstract}
Mesalamine and its derivatives are effective and well-tolerated therapies for ulcerative colitis. However, patient adherence to traditional mesalamine-based therapy is poor, and is often limited by heavy pill burdens and frequent dosing intervals. This can lead to ineffective disease control, impaired quality of life, and preventable morbidity and mortality. Previous studies have suggested that a once-daily mesalamine regimen would be strongly adhered to in the outpatient setting, but at that time no such formulation of mesalamine existed. In 2007, clinical trial data showed a novel, once-daily, multi-matrix (MMX) formulation of mesalamine to be effective in both remission induction and remission maintenance. This breakthrough in drug delivery allowed the unification of an effective therapeutic with a formulation that enables outpatients to be increasingly adherent to their medication. In theory, this might result in improved outpatient disease control and a decreased number of flares. As the use of MMX mesalamine increases, studies examining the outpatient community adherence rate need to be performed.
\end{abstract}

Keywords: mesalamine, MMX, Lialda ${ }^{\mathrm{TM}}$, ulcerative colitis, inflammatory bowel disease, adherence

\section{Introduction to management issues in ulcerative colitis, patient considerations}

Ulcerative colitis (UC) is a chronic inflammatory bowel disorder of indeterminate etiology characterized by inflammation limited to the colonic mucosa. The disease can affect small portions, extensive segments, or the entirety of the large intestine. Symptoms can include diarrhea that may be bloody, abdominal pain, cramping, as well as numerous "extra-intestinal" manifestations. The natural history of UC is one of chronic relapse and remission, with a disease course marked by periods of relative quiescence punctuated by occasional flares. UC flares are typified by acute onset of increased stool frequency, low-grade fevers, grossly bloody bowel movements, and often debilitating abdominal pain. Patients with UC also have a well-described predisposition towards the development of colorectal cancer. ${ }^{1}$ It is clear from an extensive body of literature that poorly controlled UC has a significant impact on patient quality of life, with extensive associated morbidity and even mortality. ${ }^{2-4}$

Effective therapy for UC necessitates a two-pronged approach. Standard treatment includes a daily maintenance medication regimen for those patients in whom disease is quiescent. However, equally as crucial is acute "induction-of-remission" therapy for patients experiencing disease flares. For over 50 years, the 5 -aminosalicylate (5-ASA) agents have been successfully used in both maintenance-of-remission as well as in the induction-of-remission during UC flares. Even today, these drugs and their derivatives are still considered first line therapy due to their efficacy, favorable side effect and safety profiles, and relatively low cost. ${ }^{5}$ However, a longstanding obstacle 
to effective UC treatment has been the heavy pill burden mandated by 5-ASA therapy, and the resultant difficulties with adherence to treatment regimens. Historically, patients with UC flares might be expected to take up to 16 pills on a daily basis. ${ }^{6}$ Recent studies have alerted clinicians to the low levels of adherence in outpatient UC treatment, ${ }^{7,8}$ and have pushed researchers towards development of an effective yet manageable outpatient treatment paradigm.

In 2007, a once-daily formulation of oral 5-ASA, MMX mesalamine (Lialda ${ }^{\mathrm{TM}}$; Shire Pharmaceuticals Inc., Wayne, PA, USA) was approved in the USA by the Food and Drug Administration for the induction-of-remission in mild-tomoderate UC. Approval was based on multiple randomized controlled trials ${ }^{9-11}$ that demonstrated a significant response rate compared to placebo, as measured by number of patients achieving both a complete clinical and endoscopic remission of their UC flare. However, the role of MMX mesalamine in maintenance-of-remission remained unclear.

More recent follow-up data suggest that once- or twicedaily therapy with MMX mesalamine is as effective as previous 5-ASA formulations for maintenance of clinical and endoscopic remission in $\mathrm{UC}$, measured out to one year of follow-up. ${ }^{12}$ Although adherence rates in this study were impressive, they were nonetheless achieved in a controlled setting well known to artificially inflate such results. It still remains to be seen whether outpatients with UC will be equally adherent to a decreased dosing frequency. If so, this may represent a significant advance for patients whose adherence to therapy, and consequently whose disease control, is tied to lower pill burden and simpler dosing regimens.

\section{Review of formulation, pharmacology, mode of action, pharmacokinetics of MMX mesalamine}

MMX mesalamine is a novel formulation of mesalamine that delivers a large dose of 5-ASA (1.2 g per pill) in a unique multimatrix system (MMX). The exact mechanism of action of 5-ASA is not entirely understood, but it is believed to work through downregulation of several independent pro-inflammatory cellular cascades, thereby limiting the development of intestinal inflammatory responses..$^{5}$ These pathways include, but are not limited to, the cyclooxygenase (COX) and lipoxygenase pathways, which produce inflammatory cytokines such as prostaglandins and leukotrienes. Other proposed mechanisms of 5-ASA include inhibition of both TNF-alpha as well as the intranuclear pro-inflammatory transcription factor NF-kappaB. ${ }^{13}$

The introduction of MMX technology allows improved and targeted drug delivery throughout the terminal ileum and entire colon. MMX mesalamine features an outer $\mathrm{pH}$-dependent coating that serves to protect the inner multimatrix core until exposed to a $\mathrm{pH}$ of above $7.0 .^{14-16}$ This protects the tablet core from gastric milieu and allows it to remain intact until entering the terminal ileum. There, the outer coating dissolves and exposes the core, which is composed of both hydrophilic and lipophilic matrices. When exposed to ileal intestinal fluid, the core swells up due to its hydrophilic component, forming an outer viscous gel mass, and diffusion of 5-ASA begins to occur. The diffusion rate might be too rapid to allow for drug delivery throughout the entire colon were it not for the lipophilic matrix component of the core. It is thought that the lipophilic matrix slows the entrance of water to the tablet core, resulting in a constantly evolving surface gel mass and slower diffusion of 5-ASA from the tablet core to the surface, where it eventually breaks off into small pieces, delivering 5-ASA to the colonic mucosa. ${ }^{14-16}$

Pharmacokinetic studies have shown that approximately $80 \%$ of the 5 -ASA in MMX mesalamine is absorbed in the colon, while the remaining $20 \%$ is absorbed in the terminal ileum. ${ }^{15}$ Its overall bioavailability is approximately $20 \%$, similar to other 5-ASA compounds. ${ }^{17-19}$ Once absorbed, MMX mesalamine is converted to its major inactive metabolite, N-acetyl-5-ASA, by acetyltransferase enzymes in the intestinal epithelium and the liver. ${ }^{20}$ The drug is then eliminated in equal proportions by the stool, via direct secretion back into the gastrointestinal lumen, and by the kidneys, which secrete the hepatically metabolized 5-ASA into the urine. ${ }^{5,21}$ The half-life of 5-ASA is 7 to 9 hours, and consequently 4 to 5 days are required to achieve steady-state plasma concentrations.

\section{Efficacy studies, including any comparative studies}

Published in 2007, two sister clinical trials, each a Phase III, randomized, double-blinded, multi-national, placebo-controlled trial, examined the efficacy and safety of MMX mesalamine., ${ }^{9,10}$ They independently showed that MMX mesalamine, in either once- or twice-daily dosing, was effective for induction-of-remission in flares of mildto-moderate UC. An open-label follow-up study was also performed in order to examine the long-term efficacy and safety of MMX mesalamine in the maintenance of clinical and endoscopic remission.

In the first of the sister trials, Lichtenstein et al randomized 280 patients with active mild-to-moderate UC to receive placebo, MMX mesalamine $2.4 \mathrm{~g}$ per day (divided into $1.2 \mathrm{~g}$ twice-daily dosing), or MMX mesalamine $4.8 \mathrm{~g}$ per day 
(dosed in its entirety once-daily). ${ }^{9}$ Exclusion criteria included patients with severe UC, an ongoing refractory relapse lasting more than 6 weeks, evidence of prior mesalamine treatment failure, and recent steroid, other immunosuppressant, or antibiotic use. The primary endpoint was clinical and endoscopic remission after 8 weeks of therapy. Initial disease severity and remission were measured by a modified version of the Ulcerative Colitis Disease Activity Index (UC-DAI). The author's modification of the UC-DAI rendered the definition of remission even more stringent than usual, meaning that in order to have achieved remission in this study, patients needed to meet more rigid clinical and endoscopic criteria, such as three consecutive symptom-free days as well as absence of mucosal friability on sigmoidoscopy. Significantly more patients treated with MMX mesalamine $2.4 \mathrm{~g}$ per day or $4.8 \mathrm{~g}$ per day achieved both clinical and endoscopic remission at the end of 8 weeks as compared to placebo (34.1\% vs $29.2 \%$ vs $12.9 \%$, respectively). Median time to clinical remission was also significantly improved in the treatment groups. Analysis of additional secondary endpoints demonstrated significant improvements in the number of patients who experienced clinical remission or sigmoidoscopic remission alone, as well as fewer treatment failures after 8 weeks.

The second sister trial, published by Kamm et al, featured a near-identical study design, with the addition of a reference arm of patients who received conventional delayed-release oral mesalamine (Asacol ${ }^{\circledR}$; Proctor and Gamble, Cincinnati, $\mathrm{OH}, \mathrm{USA}$ ) thrice-daily. ${ }^{10}$ Also, the MMX mesalamine in this trial was dosed at either $4.8 \mathrm{~g}$ per day or $2.4 \mathrm{~g}$ per day, all delivered in one undivided daily dose. Overall, 343 patients were randomized. Inclusion and exclusion criteria, methods, primary and secondary endpoints, and measures of disease severity were essentially identical to the sister trial. This trial also revealed a significant improvement in clinical and endoscopic remission rates after 8 weeks of $2.4 \mathrm{~g}$ per day or $4.8 \mathrm{~g}$ per day of MMX mesalamine therapy as compared to placebo $(40.5 \%, 41.2 \%$, and $22.1 \%$, respectively). Subgroup analysis confirmed a significant benefit in patients receiving MMX mesalamine regardless of severity (mild vs moderate) or extent of disease (left-sided vs pancolitis). Interestingly, the reference Asacol arm showed no significant improvement in clinical and endoscopic remission rates when compared to placebo $(32.6 \%$ vs $22.1 \%, \mathrm{p}=0.124)$. This stands in contrast to conclusions from previous literature, most notably the ASCEND trials, where Asacol was demonstrated to be an effective therapeutic in moderately active UC. ${ }^{22,23}$ It is possible that the current study was underpowered to detect a significant difference in the Asacol arm.
At the completion of the above trials, patients who did not achieve both clinical and endoscopic remission were allowed to enroll in an acute extension trial of once-daily, high dose (4.8 g per day) MMX mesalamine for an additional 8 weeks. ${ }^{24}$ Of the 304 patients who opted for this treatment, $59.5 \%$ achieved clinical and endoscopic remission after 8 weeks. This suggested that prolonged, high-dose MMX mesalamine might be an alternative to "step-up" therapies such as immunosuppressants and/or steroids.

Patients from all three studies who achieved clinical and endoscopic remission were allowed to enroll in a randomized, open-label, multicenter, one-year follow-up study to examine the ability of MMX mesalamine to maintain remission in mild-to-moderate UC. ${ }^{12}$ In addition, a number of patients $(n=89)$ who had not met the strict remission criteria, but who were deemed to be in good enough health at the conclusion of the prior studies, were also allowed to enroll. In total, this trial examined 459 patients and randomized them to receive either MMX mesalamine 2.4 g per day (given once-daily) or MMX mesalamine $2.4 \mathrm{~g}$ per day (given as $1.2 \mathrm{~g}$ twice-daily). There was no placebo group, as it was considered unethical given the clear effectiveness of 5-ASA compounds in maintaining $\mathrm{UC}$ remission. Inclusion and exclusion criteria were predefined based on the outcomes from the prior parent trials. The primary outcome measure was assessment of safety and tolerability, with a secondary objective being comparison of remission rates between the two treatment groups.

In terms of safety and tolerability, results showed no significant difference in mean duration of drug exposure (mean $=47$ weeks), number, type, or severity of adverse event, time to withdrawal, or any other measured performance characteristic such as vital signs or laboratory testing. ${ }^{12}$ Overall adverse event rates were similar to placebo rates from established trials. ${ }^{25,26}$ These results point towards a long-term safety profile for MMX mesalamine which mirrors other 5-ASA compounds.

In terms of efficacy, the year-long administration of MMX mesalamine in either once- or twice-daily dosing was equally effective in maintaining clinical and endoscopic remission $(64.4 \%$ vs $68.5 \%$ respectively, $\mathrm{p}=0.351)$. These results are in keeping with the majority of published remission rates for conventional oral 5-ASA treatments. ${ }^{22,27}$ As might be expected, results varied impressively depending on whether the patient enrolled directly from the parent trials, or whether they necessitated an additional 8 weeks of high-dose mesalamine therapy prior to achieving remission. Patients who required the additional therapy were significantly less likely to be in remission after 12 months of maintenance therapy 
(56\% vs $76 \%, \mathrm{p}<0.0001)$. This argues that patients who were more responsive to MMX mesalamine for inductionof-remission were likely to respond to MMX mesalamine when used for maintenance therapy.

Also not unexpectedly, subjects who did not meet the initial stringent remission criteria, but who were nonetheless added to the study after being deemed well enough by their doctor ("intention-to-treat" subjects), did worse. These 89 additional subjects had remission rates that were lower than the intention-to-treat group as a whole, which also included the "per-protocol" subjects. Once- or twice-daily MMX mesalamine dosing in these 89 patients did not result in a significant difference in remission rate $(52.1 \%$ vs $51.2 \%$, respectively). ${ }^{12}$

However, when the entire intention-to-treat data were compared to the per-protocol population data, there was no significant difference in remission rate. This points towards the conclusion that even patients who do not endoscopically respond to induction-of-remission therapy with MMX mesalamine, but who still achieve clinical remission and are deemed "well" by their clinician, can reasonably hope to achieve clinical and endoscopic remission when receiving 2.4 g per day of maintenance MMX mesalamine. ${ }^{12}$

\section{Safety and tolerability}

5-ASA compounds have the distinct advantage of over a half-century's worth of study and re-formulation in attempts to improve drug safety and tolerability. Initial formulations such as sulfasalazine, which contains a sulfapyridine moiety linked to the 5-ASA via an azo bond, had a higher rate of adverse events (AEs) that directly correlated with overall exposure to the sulfa moiety. ${ }^{28}$ However, sulfa-free formulations of 5-ASA are better tolerated. In fact, it has been shown that up to $90 \%$ of patients who stopped taking sulfasalazine due to its side effects were able to tolerate 5-ASA. ${ }^{5,22}$

Multiple trials, including a large meta-analysis, have demonstrated that 5-ASA preparations, including MMX mesalamine, have a rate of AEs similar to placebo., ${ }^{9,10,29,30}$ In a phase II dose-ranging study, MMX mesalamine was well-tolerated at all studied doses, with a low incidence of AEs consistent with the known safety profile of 5-ASA. ${ }^{30}$ Although this study did note a trend towards more AEs in the highest dose subgroup (4.8 g per day), no study participants discontinued treatment due to AEs. In contrast, both large phase III remission induction studies failed to show any doseresponse relationship between MMX mesalamine and AEs. Both studies found no significant difference in the rates of AEs between MMX mesalamine and placebo. The majority of AEs were mild or moderate. The most common AEs were gastrointestinal disorders, including nausea, flatulence, diarrhea, and worsening of UC, as well as other conditions such as headache.

Recent data have confirmed an interaction between 5-ASA compounds and thiopurines such as azathioprine. While the mechanism of this interaction is not entirely elucidated, concurrent administration of mesalamine and azathioprine appears to significantly increase levels of 6-thioguanine nucleotides, the major active marrow toxic derivative of azathioprine. ${ }^{31}$ Likewise, withdrawal of mesalamine from a 5-ASA and thiopurines regimen has been shown in vivo to result in significantly decreased levels of 6-thioguanine nucleotides. ${ }^{32}$ A theoretical risk of dosage toxicity does exist with the addition of MMX mesalamine to a drug regimen that includes a thiopurine, and appropriate surveillance labs and drug levels should be monitored closely.

\section{Patient-focused perspectives such as quality of life, patient satisfaction/ acceptability, adherence and uptake}

Even the most effective medical therapies in $\mathrm{UC}$ are of no utility if patients are unable to adhere to the treatment regimen. Historically, physicians have notoriously overestimated patients' rates of adherence, a misjudgment that may have dire consequences. Studies have shown that as few as $40 \%$ of patients are adherent to their traditional maintenance UC therapy. ${ }^{8}$ Not surprisingly, patients who are non-adherent to their medication (defined as filling fewer than $80 \%$ of their prescriptions) are over five times more likely to experience a disease flare as compared to adherent patients. ${ }^{7}$

Clearly, adherence to a medication regimen reduces disease activity. Disease activity in UC, in turn, has been shown to be a primary determinant of patients' general life satisfaction (GLS) and health-related life satisfaction (HRLS). ${ }^{2-4}$ Hence it is reasonable to conclude that an easily adhered-to UC regimen would directly improve patients' quality of life and overall satisfaction.

But the successful design of a UC regimen that allows adherence without compromising efficacy has not yet been described. Understanding which factors affect patient adherence is critical to designing such a regimen. Previous literature has identified male gender, taking more than four prescription medications concomitantly, and disease limited to the left side of the colon as risk factors for non-adherence. ${ }^{8}$ More recently, a survey of 1595 patients with UC receiving 5-ASA therapy showed the most common reasons for non-adherence were "too many pills," "dosing required too 
many times each day," "medication too inconvenient," and "forgetting to take medication". ${ }^{33}$ These sentiments have been echoed elsewhere. ${ }^{34,35}$ Despite having knowledge of these barriers to effective therapy, providers were still limited by the half-life and pill burden of traditionally available medications.

In an effort to demonstrate improved adherence with a smaller pill burden, a small 2003 pilot study compared oncedaily vs conventional dosing of traditional mesalamine. ${ }^{36}$ A significant difference in patient adherence was observed (100\% vs $70 \%$ ). Interestingly, once-daily dosing had the same rate of disease relapse as the conventional dosing group. However, this study was neither designed nor powered to evaluate the efficacy of once-daily dosing, but rather simply to monitor adherence. It confirmed that outpatients with UC are better able to adhere to a once-daily regimen as compared to a regimen with more frequent dosing. However, a major obstacle remained the lack of an available drug that combined this dosing frequency with proven efficacy.

When phase III clinical trial data demonstrated that oncedaily MMX mesalamine was effective in inducing remission of active $\mathrm{UC}$, it provided hope that this convenient regimen might also be effective in maintaining disease quiescence. This would unite the benefits of clinical efficacy and ease-ofuse for long-term therapy. As discussed above, one year follow-up data have shown MMX mesalamine to be effective in maintenance of remission. That study reported that $96 \%$ of patients enrolled were adherent to their medication regimen, having taken more than $80 \%$ of their prescribed medication. $^{12}$

While this may appear to be an exciting step towards realizing the "holy grail" of medical therapy, it remains to be seen if such adherence rates are generalizable to the community setting. The $96 \%$ adherence rate was obtained in a strictly monitored clinical setting, which is well known to produce unrealistic adherence rates. However, as seen in the 2003 pilot study of once-daily mesalamine dosing, adherence in the community setting with a once-daily mesalamine regimen did yield significantly improved adherence when compared to traditional outpatient regimens. As the use of MMX mesalamine increases, more studies of community adherence need to be performed in order to evaluate for any significant change from previous rates.

\section{Conclusions, place in therapy}

Overall, the existing data support MMX mesalamine as a safe and effective once-daily medication for induction and maintenance of remission in mild-to-moderate ulcerative colitis. Its potential for clinical use is not yet fully realized, and may still be limited by hesitation from physicians, patients, and the insurance industry. ${ }^{37}$ Certainly, placebo controlled studies directly comparing MMX mesalamine and other 5-ASA compounds dosed in a once-daily manner must be done in order to establish non-inferiority in the maintenance setting. Additionally, community adherence studies must be performed to evaluate the prospect of MMX mesalamine as a breakthrough in convenience and potential for adherence. However, initial studies provide compelling evidence that MMX mesalamine may represent the first once-daily oral 5-ASA therapeutic that provides the clinical efficacy and safety patients expect, while also eliminating the pill burden and inconveniences that have traditionally represented formidable barriers to adherence and effective therapy.

\section{Disclosures}

Dr. Zandman has no disclosures. Dr. Peppercorn is a member of the speaker's bureau for Abbott Loboratories and for Proctor \& Gamble.

\section{References}

1. Eaden JA, Abrams KR, Mayberry JF. The risk of colorectal cancer in ulcerative colitis: a meta-analysis. Gut. 2001;48(4):526-535.

2. Casellas F, López-Vivancos J, Badia X, Vilaseca J, Malagelada JR. Influence of inflammatory bowel disease on different dimensions of quality of life. Eur J Gastroenterol Hepatol. 2001;13(5):567-572.

3. Casellas F, Arenas JI, Baudet JS, et al. Impairment of health-related quality of life in patients with inflammatory bowel disease: a Spanish multicenter study. Inflamm Bowel Dis. 2005;11(5):488-496.

4. Janke KH, Klump B, Gregor M, Meisner C, Haeuser W. Determinants of life satisfaction in inflammatory bowel disease. Inflamm Bowel Dis. 2005;11(3):272-286.

5. Nielsen OH, Munck LK. Drug insight: aminosalicylates for the treatment of IBD. Nat Clin Pract Gastroenterol Hepatol. 2007;4(3):160-170.

6. Hu MY, Peppercorn MA. MMX mesalamine: a novel high-dose, oncedaily 5-aminosalicylate formulation for the treatment of ulcerative colitis. Expert Opin Pharmacother. 2008;9(6):1049-1058.

7. Kane S, Huo D, Aikens J, Hanauer S. Medication nonadherence and the outcomes of patients with quiescent ulcerative colitis. Am J Med. 2003;114(1):39-43.

8. Kane SV, Cohen RD, Aikens JE, Hanauer SB. Prevalence of nonadherence with maintenance mesalamine in quiescent ulcerative colitis. Am J Gastroenterol. 2001;96(10):2929-2933.

9. Lichtenstein GR, Kamm MA, Boddu P, et al. Effect of once- or twicedaily MMX mesalamine (SPD476) for the induction of remission of mild to moderately active ulcerative colitis. Clin Gastroenterol Hepatol. 2007;5(1):95-102

10. Kamm MA, Sandborn WJ, Gassull M, et al. Once-daily high concentration MMX mesalamine in active ulcerative colitis. Gastroenterology. 2007;132(1):66-75

11. Sandborn WJ, Kamm MA, Lichtenstein GR, Lyne A, Butler T, Joseph RE. Multi Matrix System mesalazine for the induction of remission in patients with mild-to-moderate ulcerative colitis: a combined analysis of two randomized, double-blind, placebo-controlled trials. Aliment Pharmacol Ther. 2007;26(2):205-215.

12. Kamm MA, Lichtenstein GR, Sandborn WJ, et al. Randomized trial of once- or twice-daily MMX mesalazine for maintenance of remission in ulcerative colitis. Gut. 2008;57(7):893-902. 
13. Kaiser GC, Yan F, Polk DB. Mesalamine blocks tumor necrosis factor growth inhibition and nuclear factor kappaB activation in mouse colonocytes. Gastroenterology. 1999;116(3):602-609.

14. Baker DE. MMX Mesalamine. Rev Gastroenterol Disord. 2006;6(4): 146-152.

15. Brunner M, Assandri R, Kletter K, et al. Gastrointestinal transit and 5-ASA release from a new mesalazine extended-release formulation. Aliment Pharmacol Ther. 2003;17(3):395-402.

16. Prantera C, Viscido A, Biancone L, Francavilla A, Giglio L, Campieri M. A new oral delivery system for 5-ASA: preliminary clinical findings for MMx. Inflamm Bowel Dis. 2005;11(5):421-427.

17. Hussain FN, Ajjan RA, Kapur K, Moustafa M, Riley SA. Once versus divided daily dosing with delayed-release mesalazine: a study of tissue drug concentrations and standard pharmacokinetic parameters. Aliment Pharmacol Ther. 2001;15(1):53-62.

18. Corey AE, Rose GM, Conklin JD. Bioavailability of single and multiple doses of enteric-coated mesalamine and sulphasalazine. J Int Med Res. 1990;18(6):441-453.

19. Sandborn WJ, Hanauer SB. Systematic review: the pharmacokinetic profiles of oral mesalazine formulations and mesalazine pro-drugs used in the management of ulcerative colitis. Aliment Pharmacol Ther. 2003;17(1):29-42.

20. Ireland A, Priddle JD, Jewell DP. Acetylation of 5-aminosalicylic acid by isolated human colonic epithelial cells. Clin Sci (Lond). 1990;78(1):105-111.

21. Klotz, U. Clinical pharmacokinetics of sulphasalazine, its metabolites and other prodrugs of 5-aminosalicylic acid. Clin Pharmacokinet. 1985;10(4):285-302.

22. Hanauer SB, Sandborn WJ, Kornbluth A, et al. Delayed-release oral mesalamine at $4.8 \mathrm{~g} / \mathrm{day}$ ( $800 \mathrm{mg}$ tablet) for the treatment of moderately active ulcerative colitis: the ASCEND II trial. Am J Gastroenterol. 2005;100(11):2478-2485.

23. Hanauer SB, Sandborn WJ, Dallaire C, et al. Delayed-release oral mesalamine $4.8 \mathrm{~g}$ /day ( $800 \mathrm{mg}$ tablets) compared to $2.4 \mathrm{~g} /$ day (400 mg tablets) for the treatment of mildly to moderately active ulcerative colitis: The ASCEND I trial. Can J Gastroenterol. 2007;21(12):827-834.

24. Kamm MA, Lichtenstein GR, Sandborn WJ, et al. Effect of extended MMX mesalamine therapy for acute, mild-to-moderate Ulcerative Colitis. Inflamm Bowel Dis. 2009;15(1):1-8.

25. Loftus EV Jr, Kane SV, Bjorkman D. Systematic review: short-term adverse effects of 5-aminosalicylic acid agents in the treatment of ulcerative colitis. Aliment Pharmacol Ther. 2004;19(2):179-189.
26. Miner P, Hanauer S, Robinson M, Schwartz J, Arora S. Safety and efficacy of controlled-release mesalamine for maintenance of remission in ulcerative colitis. Pentasa UC Maintenance Study Group. Dig Dis Sci. 1995;40(2):296-304.

27. Sutherland L, Macdonald JK. Oral 5-aminosalicylic acid for maintenance of remission in ulcerative colitis. Cochrane Database Syst Rev. 2006;19(2):CD000544.

28. Das KM, Eastwood MA, McManus JP, Sircus W. Adverse reactions during salicylazosulfapyridine therapy and the relation with drug metabolism and acetylator phenotype. N Eng J Med. 1973;289(10): 491-495.

29. Cohen RD, Woseth DM, Thisted RA, Hanauer SB. A meta-analysis and overview of the literature on treatment options for left-sided ulcerative colitis and ulcerative proctitis. Am J Gastroenterol. 2000;95(5):1263-76. Loftus, EV. A practical perspective on ulcerative colitis: patients' needs from aminosalicylate therapies. Inflamm Bowel Dis. 2006;12(12):1107-1113.

30. D'Haens G, Hommes D, Engels J, et al. Once daily MMX mesalazine for the treatment of mild-to-moderate ulcerative colitis: a phase II, doseranging study. Aliment Pharmacol Ther. 2006;24(7):1087-1097.

31. Hande S, Wilson-Rich N, Bousvaros A, et al. 5-aminosalicylate therapy is associated with higher 6-thioguanine levels in adults and children with inflammatory bowel disease in remission on 6-mercaptopurine or azathioprine. Inflamm Bowel Dis. 2006;12(4):251-257.

32. Dewit O, Vanheuverzwyn R, Desager JP, Horsmans Y. Interaction between azathioprine and aminosalicylates: an in vivo study in patients with Crohn's disease. Aliment Pharmacol Ther. 2002;16(1):79-85.

33. Loftus, EV. A practical perspective on ulcerative colitis: patients' needs from aminosalicylate therapies. Inflamm Bowel Dis. 2006;12(12): 1107-1113.

34. Shale MJ, Riley SA. Studies of compliance with delayed-release mesalazine therapy in patients with inflammatory bowel disease. Aliment Pharmacol Ther. 2003;18(2):191-198.

35. Levy RL, Feld AD. Increasing patient adherence to gastroenterology treatment and prevention regimens. Am J Gastroenterol. 1999;94(7):1733-1742.

36. Kane S, Huo D, Magnanti K. A pilot feasibility study of once daily dosing versus conventional dosing mesalamine for maintenance of ulcerative colitis. Clin Gastroenterol Hepatol. 2003;1(3):170-173.

37. Kedia P, Cohen RD. Once-daily MMX mesalamine for the treatment of mild-to-moderate ulcerative colitis. Ther Clin Risk Manag. 2007;3(5):919-927. 